\title{
Year Date
}

National Cancer Institute

\section{Source}

National Cancer Institute. Year Date. NCI Thesaurus. Code C159612.

The year in four digit format. 